\title{
Increasing Cultural Awareness Through a Cultural Awareness Program
}

\author{
Beate Baltes \\ Walden University \\ David Hernandez \\ Walden University \\ Christina Collins \\ Walden University
}

Racial tension motivates strife and violence in the metropolitan Detroit, Michigan, area. The purpose of this study was to determine the effectiveness of a collaborative partnership, the Cultural Awareness Consortium (CAC), in making a positive impact on the attitudes of a group of diverse high school students regarding multicultural relations. The two theoretical frameworks guiding this study were Allport's intergroup contact theory and intercultural competence theory originating from International Education and International Studies. The research questions concerned whether attending the CAC for 4 months, the treatment, changed students' attitudes on multicultural relations, and whether a student's gender or ethnicity was a predictor of changes in these attitudes. A single group, pre-experimental design with data collection from two administrations of the Student Multicultural Relations Survey was used in the study. Fifty-four students completed the survey, which yielded four multicultural relations scales (dependent variables), eight single-item attitudinal variables on multicultural issues, and two demographic variables (independent variables), and inferential analysis included $t$ tests and multiple regression. According to study results, students' attitudes on multicultural relations had changed significantly, and students talked to and mixed with students from different cultural backgrounds more after the treatment. Educational institutions providing experiences like the CAC can make a positive impact on students' attitudes on multicultural relations. This can lead to positive social change as students increase their acceptance of others and take those attitudes and values with them into the workforce after they graduate, serving as role models of acceptance for their peers.

Keywords: multiculturalism, racism, tolerance

\section{Introduction}

Racial tension motivates strife and violence in the metropolitan Detroit area. The Federal Bureau of Investigation (FBI, 2010) reported 6,628 crime victims who were different to their attacker in race, religion, sexual orientation, ethnicity/national origin, or disability. In Michigan, crimes related to race, ethnicity, and national origin rose 1\%, from 219 incidents to 226 incidents, between 2009 and 2010 (FBI, 2009, 2010). In public schools, the percentage of crimes motivated by cultural and ethnic differences increased as students became older from $3 \%$ in third grade to $5 \%$ in 12 th grade (United States Census Bureau, 2012). These statistics do not include noncrimes, such as the note containing 
racist language found at Seaholm High School in Birmingham, Michigan, that disparaged an African American student and three African American teachers (Maddox, 2012).

Racial divisions exist among students, from elementary through high school, and these divisions must be addressed through cultural awareness training. One way to affirm cultural differences in the classroom might be to establish collaboratives such as the intercultural collaboration of the Cultural Awareness Consortium (CAC), which provides high school students in Michigan opportunities to interact with students from various cultures. Encouraging interactions with diverse people provides opportunities for personal and academic growth (DeLong et al., 2011). Allport (1958) reported that cultural awareness and interpersonal contact among diverse cultural groups might serve to promote intergroup relationships and acceptance. Other researchers claimed that increases in cultural awareness might enhance students' educational experiences (Carter \& Vuong, 1997; Richardson, 2012). Therefore, this study investigated the relationship between high school students' attitudes on multicultural relations and their participation in the CAC.

\section{The Cultural Awareness Consortium}

The CAC is located in an urban school district in Michigan. Students from six neighboring school districts are selected through an application process, which requires counselor recommendation, referrals from teachers, a grade point average of 3.0 or better, and an interview from the consortium board. The CAC invites students to attend afternoon classes every day for $2 \mathrm{hr}$ throughout the school year. During this time, students enroll in the advanced placement courses that are not available in their home high school. Students are integrated in these classes-they sit together, work collaboratively on projects, and engage each other in reflective activities. The policies of the CAC are designed to foster a harmonious culture within the school, regardless of ethnic group or religion, and every student is expected to show respect to school staff and fellow students. Students who are disrespectful and cannot get along with others in the CAC may lose their privilege of attending this school. The CAC students interact with students of different cultural backgrounds while being monitored by teachers to ensure a positive cross-cultural collaborative experience. The CAC provides a well-designed curriculum to deepen, explore, and engage students with daily interactions/discussions of political issues, social injustices, and cultural views. The activities and discussions consisted of sharing cultural history and personal interests. Discussions often occurred on (a) ancestry lineage, (b) movement of cultural groups across geographic locations, and (c) the parallel and vertical alignment of ethnic commonalities, which are under the umbrella of "culturally responsive pedagogy" (Richards, Brown, \& Forde, 2007, p. 64). Such discourse is thought to create a foundation for a higher level of understanding during students' peer-to-peer interactions. During these classroom curricular interactions, students' assignments of projects, activities, and collaborations required skills to recognize the similarities and differences in cultural or environmental behaviors. In an attempt to connect the diverse groups and increase cultural awareness, students may compare and contrast approaches to life, analyze and debate, as well as justify and explain them. Such interactions have more meaning than surface level interactions, and foster understanding of how people greet each other, what they talk about, and how they express themselves are characteristics of being culturally aware (Hansell, 2000; Quappe \& Cantatore, 2005; Riskowski \& Olbricht, 2010), which should lead to increased intercultural awareness.

To increase cultural awareness, Loukaitou-Sideris (2003) found that children must be given a common ground, or place to interact, play, talk, and collaborate across cultural lines. In other words, through intradistrict interactions, students will share their history, customs, and native language as they learn about each other. Reich and Reich (2006) agreed that cultural awareness promoted within the same school could allow students to better understand the diverse country and world they 
inhabit. Furthermore, former University of Michigan President Lee Bollinger argued that students "must be immersed in a campus culture that allows them to study with, argue with and become friends with students who may be different from them" (as cited in Rothman, Lipset, \& Nevitte, 2003, p. 25).

\section{Theoretical Framework}

Allport's (1958) intergroup contact theory and the theory of the intercultural competence, outlined by the International Education and International Studies organization, served as the two theoretical frameworks guiding the study.

\section{Intergroup Contact Theory}

Allport (1958) determined that interpersonal contact might be one of the most effective ways to reduce prejudice among diverse cultural groups. Allport maintained that in order for prejudice to be reduced, the collaborating members should have equal status, a common goal, and there should be no competition between the groups. Intergroup contact was defined as interactions between members of defined cultural groups who meet face-to-face (Pettigrew \& Tropp, 2006). The optimal conditions are an environment or situation in which students feel safe to speak and interact with others (Pettigrew \& Tropp, 2006).

Intergroup contact theory has been applied in the public school setting to decrease racial tension and minimize divisions between cultures by incorporating guidelines and support for students to work together in collaborative groups (Pettigrew \& Tropp, 2006; Schuitema \& Veugelers, 2011). As contact among diverse students' increases, self-awareness and opportunities to learn from others have also been shown to increase (Bazron, Osher, \& Fleishmann, 2005; Pettigrew, 1998; Slavin \& Cooper, 1999). Ultimately, the goal is acceptance of others from other racial groups.

\section{Intercultural Competence Theory}

Another theory about the relationships between diverse groups of people is the theory of intercultural competence. According to Hansell (2000), to grow as a productive adult and citizen, individuals need to depend on their "ability to successfully negotiate cultural differences and to appreciate diverse perspectives" (Intercultural Competence section, para. 1). To achieve this goal, Liaw (2006) noted that students must explore their own culture through discussion of the value system, expectations, traditions, customs, and rituals they unconsciously take part in before they are able to reflect upon other cultures with a "higher degree of intellectual objectivity" (p. 50). As the exchanges of communication between diverse groups increase, students begin to gain insight into other groups' rituals, traditions, and values. Baltes (1998) indicated that, as students understand diverse perspectives, racial relations are enhanced. Some researchers have suggested that the components of intercultural competence consist of cross-cultural awareness, empathy, and flexibility (Chen \& Starosta, 1996; Deardorff, 2004). Deardorff (2004) stated that intercultural competence "involves the development of one's skills and attitudes in successfully interacting with persons of diverse backgrounds" (p. 15). Intercultural competence is a skill learned through intergroup contact.

\section{Methodology}

A single group, pre-experimental research design was used to explore the impact of students participating for 4 months in a CAC on attitudes on multicultural relations. 
The dependent variable was measured through the Student Multicultural Relations Survey that was initially designed by Rothfarb (1992), but later revised by Woods (2009), and used with permission. The survey had 27 questions that required a Likert-style response; 26 questions used strongly agree, agree, neutral, disagree, and strongly disagree, and one question used never, rarely, often, sometimes, and always. Additionally, the survey had three questions requiring a dichotomous yes/no response and two demographic questions-gender and race/ethnicity. Because survey data were collected anonymously, the final section of the survey posed three questions (participant's day of the month of birth, first two letters of his or her street address, and middle initial) that aided in matching completed survey from the initial survey administration with the second survey administration. Content validity of the survey was established through multicultural experts (Rothfarb, 1992). Additionally, convergent (external) validity of the survey scales was established through comparison of the factors in the Rothfarb study with those factors from other similar studies. Woods (2009) reported the survey's scales' internal consistency (Cronbach's alpha) fell between .78 to .83. Finally, Woods reported the survey's Flesh-Kincaid readability index at 8.94, or slightly below ninth grade. Therefore, the Student Multicultural Relations Survey had sufficient validity, reliability, and readability to be used in this research with 10th-grade participants.

The independent variables, besides the treatment variable of participating in the CAC or not participating in the CAC, were students' gender and ethnicity. The setting for this research was a CAC located in Michigan with a culturally diverse student population of 309 students in Grades 10 to 12. Students attending the CAC were drawn from six neighboring school districts, and students had to carry a 3.0 or higher grade point average. The demographic makeup of the CAC was $65 \%$ European American, 23\% African American, 1\% Hispanic American, 5\% Asian American, and 6\% other race/ethnicity; and $66 \%$ male and $44 \%$ female.

During the first administration of the survey, 123 students completed the survey, and during the second administration, 141 students completed the survey. Pretreatment participant surveys were matched to posttreatment surveys on gender, race/ethnicity, current grade, calendar day of birth, first two numbers of student's street address, and the student's middle initial. After the matching process was completed, there were 54 usable matched pre/posttreatment surveys.

\section{Data Collection and Analysis}

The first administration of the survey was completed in September 2012 before the incoming students began the program of the CAC. The second administration of the survey was undertaken in January 2013. Descriptive statistics were calculated and reported for all variables. For demographic and survey response data measured on a nominal scales, frequencies and percentages were reported. Inferential statistical analyses were used to answer the two research questions. Repeated measures $t$ tests were used to answer the first research question.

1. Does 4 months of participation in the CAC significantly change students' attitudes on multicultural relations, as measured by the Student Multicultural Relations Survey?

$\mathrm{H}_{01}$ : There are no significant differences in students' mean multicultural relations scores as a result of participating in the CAC for 4 months.

$\mathrm{H}_{11}$ : There are significant difference in students' mean multicultural relations scores as a result of participating in the CAC for 4 months. 
Mean scores on the scales and Survey Items gathered on the first administration of the Student Multicultural Relations Survey were compared to the mean scores of the scales and Survey Items obtained from the second administration of the survey.

Multiple regression analyses were conducted to answer the second research question.

2. Does gender or ethnicity predict a change in students' attitudes on multicultural relations, as measured by the Student Multicultural Relations Survey, after 4 months of participation in the CAC?

$\mathrm{H}_{0}$ 2: $\quad$ Neither gender nor ethnicity is a significant predictor of a students' change in attitudes on multicultural relations as a result of participating in the CAC for 4 months.

$\mathrm{H}_{1} 2$ : Gender and ethnicity are significant predictors of a students' change in attitudes on multicultural relations as a result of participating in the CAC for 4 months.

The scale scores, the dependent variables, were regressed onto the gender and ethnicity variables, the independent variables. Because of the low percentage of American Indian/Native American, Asian American, and Hawaiian/Pacific Islander subgroups in the school, there was an inadequate representation of these subgroups in the data to conduct reliable inferential statistics on them. Therefore, the ethnicity variable was dummy coded into two separate variables-African American and European American. The baseline value for the dummy coded ethnicity variables was all other ethnicities than those two.

\section{Descriptive Statistics}

The participant demographics are that of the 54 respondents, $33 \%$ were male and $67 \%$ were female, with $22 \%$ being African American, $72 \%$ being European American, and 7\% being others.

Table 1 presents the frequencies and percentages of responses to survey questions with a fixedresponse, agreement Likert scale, and Table 2 presents those same descriptive statistics but for survey questions with a fixed-response, frequency Likert scale. In Table 1, there is a higher percentage of agree and strongly agree responses in the posttreatment survey data on all items except for Survey Items 15, 16, and 17. There was a positive shift in attitudes towards others from different cultural backgrounds, which was the desired result of participation in the CAC. Additionally, because Survey Item 9 was reverse coded, there was a higher percentage of disagree and strongly disagree responses, but that indicates "less fear" of students from different cultural backgrounds, which again was the desired result of participation in the CAC. 
Table 1: Frequency and Percentages for Student Responses on Survey Items 1-26 ( $N=54)$

\begin{tabular}{|c|c|c|c|c|c|c|c|c|c|c|c|}
\hline \multicolumn{5}{|c|}{ Pretreatment Survey Results } & \multirow[b]{2}{*}{$\begin{array}{c}\text { Item } \\
\#\end{array}$} & \multirow[b]{2}{*}{ Survey Question Stem } & \multicolumn{5}{|c|}{ Posttreatment Survey Results } \\
\hline $\begin{array}{l}\text { Strongly } \\
\text { Disagree }\end{array}$ & Disagree & Neutral & Agree & $\begin{array}{l}\text { Strongly } \\
\text { Agree }\end{array}$ & & & $\begin{array}{l}\text { Strongly } \\
\text { Disagree }\end{array}$ & Disagree & Neutral & Agree & $\begin{array}{l}\text { Strongly } \\
\text { Agree }\end{array}$ \\
\hline & & $\begin{array}{c}15 \\
-27.80 \%\end{array}$ & $\begin{array}{c}23 \\
-42.60 \%\end{array}$ & $\begin{array}{c}16 \\
-29.60 \%\end{array}$ & 1 & $\begin{array}{l}\text { I like to be with people who have a } \\
\text { different cultural background than } \\
\text { mine. }\end{array}$ & & & $\begin{array}{c}8 \\
-14.80 \%\end{array}$ & $\begin{array}{c}21 \\
-38.90 \%\end{array}$ & $\begin{array}{c}25 \\
-46.30 \%\end{array}$ \\
\hline & & $\begin{array}{c}10 \\
-18.50 \%\end{array}$ & $\begin{array}{c}21 \\
-38.90 \%\end{array}$ & $\begin{array}{c}23 \\
-42.60 \%\end{array}$ & 2 & $\begin{array}{l}\text { I enjoy having friends from other cultural } \\
\text { groups. }\end{array}$ & & & $\begin{array}{c}5 \\
-9.30 \%\end{array}$ & $\begin{array}{c}21 \\
-38.90 \%\end{array}$ & $\begin{array}{c}28 \\
-51.90 \%\end{array}$ \\
\hline & $\begin{array}{c}1 \\
-1.90 \%\end{array}$ & $\begin{array}{c}10 \\
-18.50 \%\end{array}$ & $\begin{array}{c}20 \\
-37.00 \%\end{array}$ & $\begin{array}{c}23 \\
-42.60 \%\end{array}$ & 3 & $\begin{array}{l}\text { I like living in a city with people from } \\
\text { many different cultural groups. }\end{array}$ & & $\begin{array}{l}2 \\
-3.70 \%\end{array}$ & $\begin{array}{c}11 \\
-20.40 \%\end{array}$ & $\begin{array}{c}16 \\
-29.60 \%\end{array}$ & $\begin{array}{c}25 \\
-46.30 \%\end{array}$ \\
\hline & $\begin{array}{c}1 \\
-1.90 \%\end{array}$ & $\begin{array}{c}25 \\
-46.30 \%\end{array}$ & $\begin{array}{l}20 \\
-37.00 \%\end{array}$ & $\begin{array}{c}8 \\
-14.80 \%\end{array}$ & 4 & $\begin{array}{l}\text { My friends like living in a city with many } \\
\text { different cultural groups. }\end{array}$ & & $\begin{array}{c}3 \\
-5.60 \%\end{array}$ & $\begin{array}{c}19 \\
-35.20 \%\end{array}$ & $\begin{array}{c}16 \\
-29.60 \%\end{array}$ & $\begin{array}{c}16 \\
-29.60 \%\end{array}$ \\
\hline \multirow[t]{3}{*}{$\begin{array}{c}3 \\
-5.60 \%\end{array}$} & $\begin{array}{c}6 \\
-11.10 \%\end{array}$ & $\begin{array}{c}23 \\
-42.60 \%\end{array}$ & $\begin{array}{c}11 \\
-20.40 \%\end{array}$ & $\begin{array}{c}11 \\
-20.40 \%\end{array}$ & 5 & $\begin{array}{l}\text { My parents encourage me to have friends } \\
\text { from other cultural backgrounds. }\end{array}$ & $\begin{array}{l}3 \\
-5.60 \%\end{array}$ & $\begin{array}{c}3 \\
-5.60 \%\end{array}$ & $\begin{array}{c}14 \\
-25.90 \%\end{array}$ & $\begin{array}{c}21 \\
-38.90 \%\end{array}$ & $\begin{array}{c}13 \\
-24.10 \%\end{array}$ \\
\hline & & $\begin{array}{c}11 \\
-20.40 \%\end{array}$ & $\begin{array}{c}21 \\
-38.90 \%\end{array}$ & $\begin{array}{c}22 \\
-40.70 \%\end{array}$ & 6 & $\begin{array}{l}\text { It is important for Americans to learn } \\
\text { foreign languages. }\end{array}$ & & $\begin{array}{l}2 \\
-3.70 \%\end{array}$ & $\begin{array}{c}9 \\
-16.70 \%\end{array}$ & $\begin{array}{c}15 \\
-27.80 \%\end{array}$ & $\begin{array}{c}28 \\
-51.90 \%\end{array}$ \\
\hline & $\begin{array}{c}7 \\
-13.00 \%\end{array}$ & $\begin{array}{c}16 \\
-29.60 \%\end{array}$ & $\begin{array}{c}22 \\
-40.70 \%\end{array}$ & $\begin{array}{c}9 \\
-16.70 \%\end{array}$ & 7 & $\begin{array}{l}\text { Students in this school usually "hang out" } \\
\text { with students of same cultural } \\
\text { background. }\end{array}$ & & $\begin{array}{c}6 \\
-11.10 \%\end{array}$ & $\begin{array}{c}21 \\
-38.90 \%\end{array}$ & $\begin{array}{c}17 \\
-31.50 \%\end{array}$ & $\begin{array}{c}10 \\
-18.50 \%\end{array}$ \\
\hline $\begin{array}{c}3 \\
-5.60 \%\end{array}$ & $\begin{array}{c}1 \\
-1.90 \%\end{array}$ & $\begin{array}{c}17 \\
-31.50 \%\end{array}$ & $\begin{array}{c}28 \\
-51.90 \%\end{array}$ & $\begin{array}{c}5 \\
-9.30 \%\end{array}$ & 8 & $\begin{array}{l}\text { In this school, students mix easily with } \\
\text { students who have different cultural ... }\end{array}$ & & $\begin{array}{c}4 \\
-7.40 \%\end{array}$ & $\begin{array}{c}8 \\
-14.80 \%\end{array}$ & $\begin{array}{c}29 \\
-53.70 \%\end{array}$ & $\begin{array}{c}13 \\
-24.10 \%\end{array}$ \\
\hline \multirow[t]{2}{*}{$\begin{array}{c}8 \\
-14.80 \%\end{array}$} & $\begin{array}{c}26 \\
-48.10 \%\end{array}$ & $\begin{array}{c}15 \\
-27.80 \%\end{array}$ & $\begin{array}{c}5 \\
-9.30 \%\end{array}$ & & 9 & $\begin{array}{l}\text { Students in this school are afraid of } \\
\text { students who have different cultural ... }\end{array}$ & $\begin{array}{c}18 \\
-33.30 \%\end{array}$ & $\begin{array}{c}25 \\
-46.30 \%\end{array}$ & $\begin{array}{c}6 \\
-11.10 \%\end{array}$ & $\begin{array}{l}2 \\
-3.70 \%\end{array}$ & $\begin{array}{c}3 \\
-5.60 \%\end{array}$ \\
\hline & $\begin{array}{c}1 \\
-1.90 \%\end{array}$ & $\begin{array}{c}5 \\
-9.30 \%\end{array}$ & $\begin{array}{c}28 \\
-51.90 \%\end{array}$ & $\begin{array}{c}20 \\
-37.00 \%\end{array}$ & 10 & $\begin{array}{l}\text { In this school, discipline is enforced fairly } \\
\text { among students regardless of their ... }\end{array}$ & & & $\begin{array}{c}5 \\
-9.30 \%\end{array}$ & $\begin{array}{c}25 \\
-46.30 \%\end{array}$ & $\begin{array}{c}24 \\
-44.40 \%\end{array}$ \\
\hline $\begin{array}{c}1 \\
-1.90 \%\end{array}$ & $\begin{array}{c}3 \\
-5.60 \%\end{array}$ & $\begin{array}{c}15 \\
-27.80 \%\end{array}$ & $\begin{array}{c}18 \\
-33.30 \%\end{array}$ & $\begin{array}{c}17 \\
-31.50 \%\end{array}$ & 11 & $\begin{array}{l}\text { In this school, teachers deal with any } \\
\text { racial or ethnic insults immediately. }\end{array}$ & & $\begin{array}{c}2 \\
-3.70 \%\end{array}$ & $\begin{array}{c}14 \\
-25.90 \%\end{array}$ & $\begin{array}{c}23 \\
-42.60 \%\end{array}$ & $\begin{array}{c}15 \\
-27.80 \%\end{array}$ \\
\hline \multirow[t]{3}{*}{$\begin{array}{c}1 \\
-1.90 \%\end{array}$} & $\begin{array}{l}2 \\
-3.70 \%\end{array}$ & $\begin{array}{c}10 \\
-18.50 \%\end{array}$ & $\begin{array}{c}31 \\
-57.40 \%\end{array}$ & $\begin{array}{c}10 \\
-18.50 \%\end{array}$ & 12 & $\begin{array}{l}\text { The students in this school respect the } \\
\text { cultural differences of other students. }\end{array}$ & & $\begin{array}{l}2 \\
-3.70 \%\end{array}$ & $\begin{array}{c}6 \\
-11.10 \%\end{array}$ & $\begin{array}{c}28 \\
-51.90 \%\end{array}$ & $\begin{array}{c}18 \\
-33.30 \%\end{array}$ \\
\hline & & $\begin{array}{c}10 \\
-18.50 \%\end{array}$ & $\begin{array}{c}22 \\
-40.70 \%\end{array}$ & $\begin{array}{c}22 \\
-40.70 \%\end{array}$ & 13 & $\begin{array}{l}\text { Teachers in this school respect the } \\
\text { cultural differences among students. }\end{array}$ & & & $\begin{array}{c}6 \\
-11.10 \%\end{array}$ & $\begin{array}{c}24 \\
-44.40 \%\end{array}$ & $\begin{array}{c}24 \\
-44.40 \%\end{array}$ \\
\hline & & $\begin{array}{c}7 \\
-13.00 \%\end{array}$ & $\begin{array}{c}24 \\
-44.40 \%\end{array}$ & $\begin{array}{c}23 \\
-42.60 \%\end{array}$ & 14 & $\begin{array}{l}\text { Administrators in this school respect } \\
\text { cultural differences among students. }\end{array}$ & & & $\begin{array}{c}5 \\
-9.30 \%\end{array}$ & $\begin{array}{c}22 \\
-40.70 \%\end{array}$ & $\begin{array}{c}27 \\
-50.00 \%\end{array}$ \\
\hline
\end{tabular}




\begin{tabular}{|c|c|c|c|c|c|c|c|c|c|c|c|}
\hline \multicolumn{5}{|c|}{ Pretreatment Survey Results } & \multirow[b]{2}{*}{$\begin{array}{c}\text { Item } \\
\# \\
\end{array}$} & \multirow[b]{2}{*}{ Survey Question Stem } & \multicolumn{5}{|c|}{ Posttreatment Survey Results } \\
\hline $\begin{array}{l}\text { Strongly } \\
\text { Disagree } \\
\end{array}$ & Disagree & Neutral & Agree & $\begin{array}{l}\text { Strongly } \\
\text { Agree }\end{array}$ & & & $\begin{array}{l}\text { Strongly } \\
\text { Disagree } \\
\end{array}$ & Disagree & Neutral & Agree & $\begin{array}{c}\text { Strongly } \\
\text { Agree } \\
\end{array}$ \\
\hline \multirow{5}{*}{$\begin{array}{c}1 \\
-1.90 \%\end{array}$} & $\begin{array}{c}2 \\
-3.70 \%\end{array}$ & $\begin{array}{c}5 \\
-9.30 \%\end{array}$ & $\begin{array}{c}22 \\
-40.70 \%\end{array}$ & $\begin{array}{c}25 \\
-46.30 \%\end{array}$ & 15 & $\begin{array}{l}\text { I try to understand customs of diverse } \\
\text { cultures. }\end{array}$ & & $\begin{array}{c}2 \\
-3.70 \%\end{array}$ & $\begin{array}{c}6 \\
-11.10 \%\end{array}$ & $\begin{array}{c}26 \\
-48.10 \%\end{array}$ & $\begin{array}{c}20 \\
-37.00 \%\end{array}$ \\
\hline & $\begin{array}{c}3 \\
-5.60 \%\end{array}$ & $\begin{array}{c}15 \\
-27.80 \%\end{array}$ & $\begin{array}{c}16 \\
-29.60 \%\end{array}$ & $\begin{array}{c}20 \\
-37.00 \%\end{array}$ & 16 & $\begin{array}{l}\text { I look for opportunities to interact with } \\
\text { different cultures. }\end{array}$ & & 3 & 14 & 20 & 17 \\
\hline & $\begin{array}{c}2 \\
-3.70 \%\end{array}$ & $\begin{array}{c}13 \\
-24.10 \%\end{array}$ & $\begin{array}{c}17 \\
-31.50 \%\end{array}$ & $\begin{array}{c}21 \\
-38.90 \%\end{array}$ & 17 & $\begin{array}{l}\text { I would welcome an opportunity to } \\
\text { participate in a foreign exchange } \\
\text { program. }\end{array}$ & $\begin{array}{c}2 \\
-3.70 \%\end{array}$ & $\begin{array}{c}-5.60 \% \\
5 \\
-9.30 \%\end{array}$ & $\begin{array}{c}-25.90 \% \\
8 \\
-14.80 \%\end{array}$ & $\begin{array}{c}-37.00 \% \\
18 \\
-33.30 \%\end{array}$ & $\begin{array}{c}-31.50 \% \\
21 \\
-38.90 \%\end{array}$ \\
\hline & $\begin{array}{c}2 \\
-3.70 \%\end{array}$ & $\begin{array}{c}13 \\
-24.10 \%\end{array}$ & $\begin{array}{c}19 \\
-35.20 \%\end{array}$ & $\begin{array}{c}20 \\
-37.00 \%\end{array}$ & 18 & I enjoy hearing different languages. & $\begin{array}{c}1 \\
-1.90 \%\end{array}$ & $\begin{array}{c}2 \\
-3.70 \%\end{array}$ & $\begin{array}{c}9 \\
-16.70 \%\end{array}$ & $\begin{array}{c}19 \\
-35.20 \%\end{array}$ & $\begin{array}{c}23 \\
-42.60 \%\end{array}$ \\
\hline & & $\begin{array}{c}9 \\
-16.70 \%\end{array}$ & $\begin{array}{c}21 \\
-38.90 \%\end{array}$ & $\begin{array}{c}24 \\
-44.40 \%\end{array}$ & 19 & $\begin{array}{l}\text { I would enjoy studying with students who } \\
\text { are from other countries. }\end{array}$ & & $\begin{array}{c}1 \\
-1.90 \%\end{array}$ & $\begin{array}{c}6 \\
-11.10 \%\end{array}$ & $\begin{array}{c}22 \\
-40.70 \%\end{array}$ & $\begin{array}{c}25 \\
-46.30 \%\end{array}$ \\
\hline \multirow[t]{7}{*}{$\begin{array}{c}4 \\
-7.40 \%\end{array}$} & $\begin{array}{c}1 \\
-1.90 \%\end{array}$ & $\begin{array}{c}14 \\
-25.90 \%\end{array}$ & $\begin{array}{c}15 \\
-27.80 \%\end{array}$ & $\begin{array}{c}20 \\
-37.00 \%\end{array}$ & 20 & $\begin{array}{l}\text { Citizens who have different national } \\
\text { backgrounds should be } \\
\text { encouraged ... }\end{array}$ & $\begin{array}{c}3 \\
-5.60 \%\end{array}$ & $\begin{array}{c}3 \\
-5.60 \%\end{array}$ & $\begin{array}{c}10 \\
-18.50 \%\end{array}$ & $\begin{array}{c}15 \\
-27.80 \%\end{array}$ & $\begin{array}{c}23 \\
-42.60 \%\end{array}$ \\
\hline & & $\begin{array}{c}14 \\
-25.90 \%\end{array}$ & $\begin{array}{c}23 \\
-42.60 \%\end{array}$ & $\begin{array}{c}17 \\
-31.50 \%\end{array}$ & 21 & $\begin{array}{l}\text { Various ethnic groups raise their children } \\
\text { differently. }\end{array}$ & & & $\begin{array}{c}6 \\
-11.10 \%\end{array}$ & $\begin{array}{c}28 \\
-51.90 \%\end{array}$ & $\begin{array}{c}20 \\
-37.00 \%\end{array}$ \\
\hline & $\begin{array}{c}7 \\
-13.00 \%\end{array}$ & $\begin{array}{c}13 \\
-24.10 \%\end{array}$ & $\begin{array}{c}25 \\
-46.30 \%\end{array}$ & $\begin{array}{c}9 \\
-16.70 \%\end{array}$ & 22 & $\begin{array}{l}\text { Students who do not speak English should } \\
\text { be given an opportunity to be... }\end{array}$ & $\begin{array}{c}1 \\
-1.90 \%\end{array}$ & $\begin{array}{c}5 \\
-9.30 \%\end{array}$ & $\begin{array}{c}19 \\
-35.20 \%\end{array}$ & $\begin{array}{c}13 \\
-24.10 \%\end{array}$ & $\begin{array}{c}16 \\
-29.60 \%\end{array}$ \\
\hline & $\begin{array}{c}3 \\
-5.60 \%\end{array}$ & $\begin{array}{c}9 \\
-16.70 \%\end{array}$ & $\begin{array}{c}22 \\
-40.70 \%\end{array}$ & $\begin{array}{c}20 \\
-37.00 \%\end{array}$ & 23 & $\begin{array}{l}\text { Having students from different ethnic } \\
\text { groups does not pose problem in classes. }\end{array}$ & $\begin{array}{c}1 \\
-1.90 \%\end{array}$ & $\begin{array}{c}2 \\
-3.70 \%\end{array}$ & $\begin{array}{c}4 \\
-7.40 \%\end{array}$ & $\begin{array}{c}25 \\
-46.30 \%\end{array}$ & $\begin{array}{c}22 \\
-40.70 \%\end{array}$ \\
\hline & $\begin{array}{c}2 \\
-3.70 \%\end{array}$ & $\begin{array}{c}14 \\
-25.90 \%\end{array}$ & $\begin{array}{c}27 \\
-50.00 \%\end{array}$ & $\begin{array}{c}11 \\
-20.40 \%\end{array}$ & 24 & $\begin{array}{l}\text { Stressing ethnic customs and traditions in } \\
\text { public school helps students ... }\end{array}$ & $\begin{array}{c}1 \\
-1.90 \%\end{array}$ & $\begin{array}{c}1 \\
-1.90 \%\end{array}$ & $\begin{array}{c}10 \\
-18.50 \%\end{array}$ & $\begin{array}{c}28 \\
-51.90 \%\end{array}$ & $\begin{array}{c}14 \\
-25.90 \%\end{array}$ \\
\hline & $\begin{array}{c}1 \\
-1.90 \%\end{array}$ & $\begin{array}{c}13 \\
-24.10 \%\end{array}$ & $\begin{array}{c}29 \\
-53.70 \%\end{array}$ & $\begin{array}{c}11 \\
-20.40 \%\end{array}$ & 25 & $\begin{array}{l}\text { Teachers should provide lessons on the } \\
\text { contributions of different ethnic groups. }\end{array}$ & & $\begin{array}{c}3 \\
-5.60 \%\end{array}$ & $\begin{array}{c}13 \\
-24.10 \%\end{array}$ & $\begin{array}{c}19 \\
-35.20 \%\end{array}$ & $\begin{array}{c}19 \\
-35.20 \%\end{array}$ \\
\hline & & $\begin{array}{c}4 \\
-7.40 \%\end{array}$ & $\begin{array}{c}14 \\
-25.90 \%\end{array}$ & $\begin{array}{c}36 \\
-66.70 \%\end{array}$ & 26 & $\begin{array}{l}\text { Diversity should be respected in our } \\
\text { schools. }\end{array}$ & & & $\begin{array}{c}2 \\
-3.70 \%\end{array}$ & $\begin{array}{c}14 \\
-25.90 \%\end{array}$ & $\begin{array}{c}38 \\
-70.40 \%\end{array}$ \\
\hline
\end{tabular}


In Table 2, there is a higher percentage of agree and strongly agree responses in the posttreatment survey data for Items $27 \mathrm{a}$ and $27 \mathrm{~b}$, but a higher percentage of disagree and strongly disagree responses for Item 27c. This means that in the CAC, both in class and during before and after school extracurricular activities on campus, students talked to or mixed with students of other cultural groups. However, students were less likely to talk to or mix with students of other cultural groups socially outside of the CAC.

Table 2. Frequency and Percentages for Student Responses on Survey Item 27: How Often Do You Talk to or Mix With Students of Other Cultural Groups?

\begin{tabular}{|c|c|c|c|c|c|c|c|c|c|c|c|}
\hline \multicolumn{5}{|c|}{ Pretreatment Survey Results } & \multirow[b]{2}{*}{$\begin{array}{c}\text { Item } \\
\#\end{array}$} & \multirow[b]{2}{*}{ Survey Question Stem } & \multicolumn{5}{|c|}{ Posttreatment Survey Results } \\
\hline $\begin{array}{l}\text { Strongly } \\
\text { Disagree }\end{array}$ & Disagree & Neutral & Agree & $\begin{array}{l}\text { Strongly } \\
\text { Agree }\end{array}$ & & & $\begin{array}{l}\text { Strongly } \\
\text { Disagree }\end{array}$ & Disagree & Neutral & Agree & $\begin{array}{l}\text { Strongly } \\
\text { Agree }\end{array}$ \\
\hline $\begin{array}{c}3 \\
-5.70 \%\end{array}$ & $\begin{array}{c}5 \\
-9.40 \%\end{array}$ & $\begin{array}{c}13 \\
-24.50 \%\end{array}$ & $\begin{array}{c}21 \\
-39.60 \%\end{array}$ & $\begin{array}{c}11 \\
-20.80 \%\end{array}$ & a. & In daily classes & $\begin{array}{c}1 \\
-1.90 \%\end{array}$ & $\begin{array}{c}2 \\
-3.80 \%\end{array}$ & $\begin{array}{c}11 \\
-20.80 \%\end{array}$ & $\begin{array}{c}18 \\
-34.00 \%\end{array}$ & $\begin{array}{c}21 \\
-39.60 \%\end{array}$ \\
\hline $\begin{array}{c}3 \\
-5.70 \%\end{array}$ & $\begin{array}{c}5 \\
-9.40 \%\end{array}$ & $\begin{array}{c}22 \\
-41.50 \%\end{array}$ & $\begin{array}{c}16 \\
-30.20 \%\end{array}$ & $\begin{array}{c}7 \\
-13.20 \%\end{array}$ & b. & $\begin{array}{l}\text { Before/after school in } \\
\text { extracurricular } \\
\text { activities }\end{array}$ & $\begin{array}{c}3 \\
-5.70 \%\end{array}$ & $\begin{array}{c}6 \\
-11.30 \%\end{array}$ & $\begin{array}{c}17 \\
-32.10 \%\end{array}$ & $\begin{array}{c}10 \\
-18.90 \%\end{array}$ & $\begin{array}{c}17 \\
-32.10 \%\end{array}$ \\
\hline $\begin{array}{c}3 \\
-5.80 \%\end{array}$ & $\begin{array}{c}4 \\
-7.70 \%\end{array}$ & $\begin{array}{c}17 \\
-32.70 \%\end{array}$ & $\begin{array}{c}16 \\
-30.80 \%\end{array}$ & $\begin{array}{c}12 \\
-23.10 \%\end{array}$ & c. & $\begin{array}{l}\text { Socially (for example, going to } \\
\text { the movies, parties, etc.) }\end{array}$ & $\begin{array}{c}5 \\
-9.40 \%\end{array}$ & $\begin{array}{c}9 \\
-17.00 \%\end{array}$ & $\begin{array}{c}12 \\
-22.60 \%\end{array}$ & $\begin{array}{c}15 \\
-28.30 \%\end{array}$ & $\begin{array}{c}12 \\
-22.60 \%\end{array}$ \\
\hline
\end{tabular}

Note. For pretreatment survey results, $N=52$; for posttreatment survey results, $N=53$. 
The responses to Survey Items 28 through 30 are presented in Figures 1, 2, and 3. According to the posttreatment data, after 4 months of participation in the CAC, more students have had friends of a different culture in their homes (see Figure 1) and/or have worked on a school or community project with students of other cultures (see Figure 2). On the other hand, after 4 months of participation in the CAC, there was absolutely no difference in students' willingness to help a member of a different cultural group with homework or tutoring (see Figure 3).

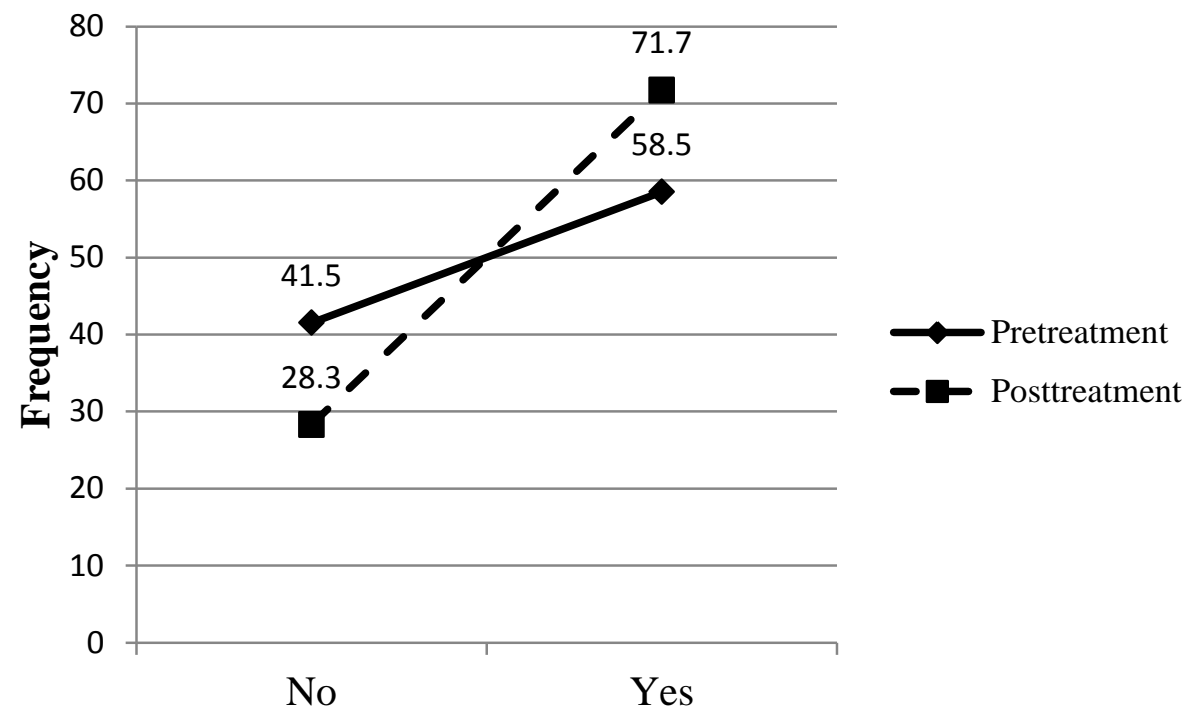

Figure 1: Frequency of Yes/No Responses for Pre/Posttreatment Data for Survey Question 28 ("I Have Had Friends of a Different Culture in My Home in the Past 12 Months") 


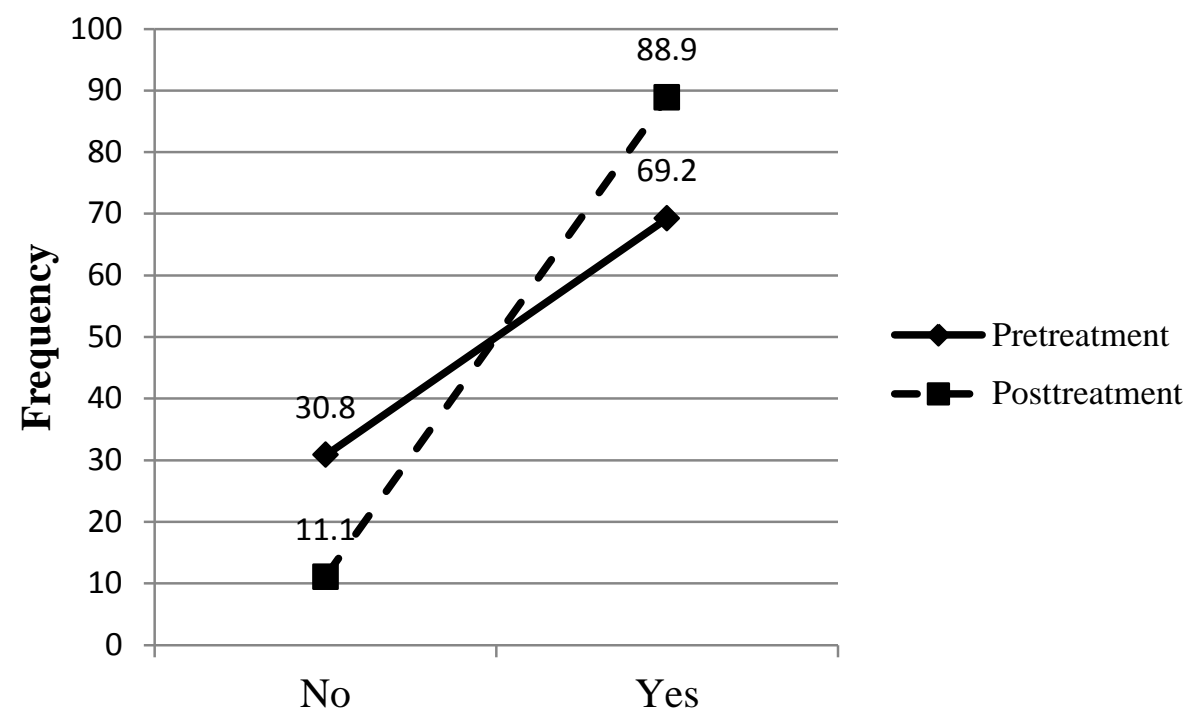

Figure 2: Frequency of Yes/No Responses for Pre/Posttreatment Data for Survey Question 29 ("I Have Worked on a School or Community Project With Students of Other Cultures in the Past 12 Months")

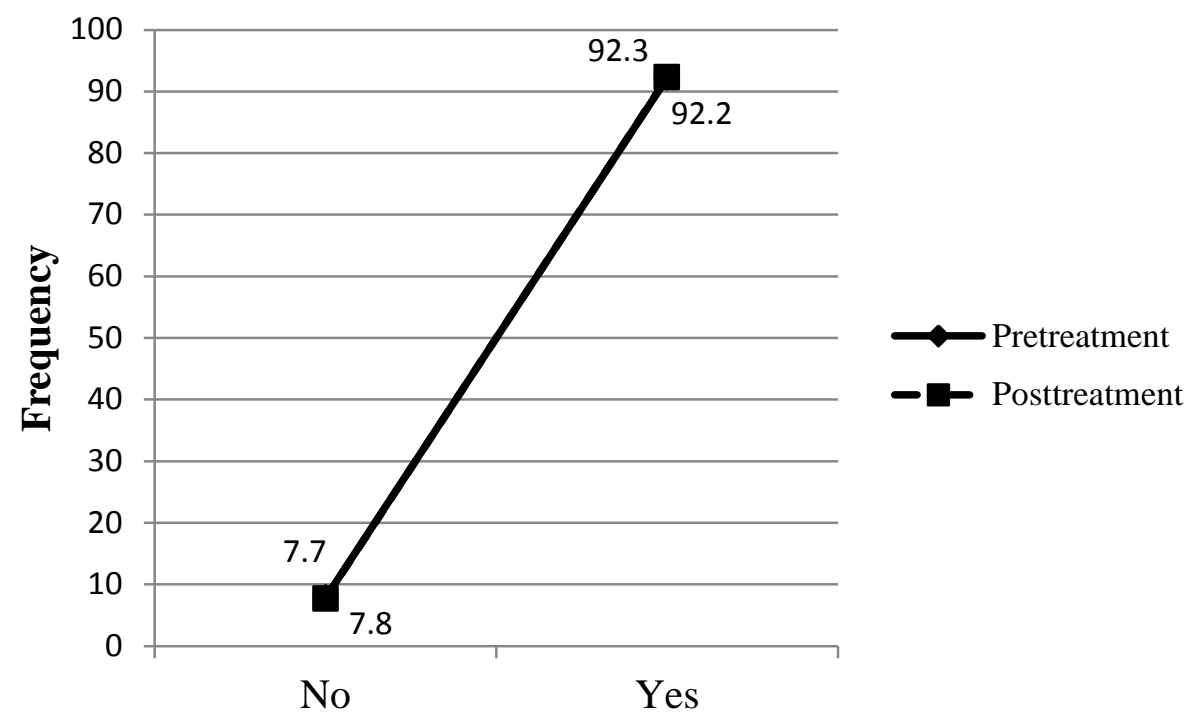

Figure 3: Frequency of Yes/No Responses for Pre/Posttreatment Data for Survey Question 30 ("I Would Be Willing to Help a Member of a Different Cultural Group With Homework or Tutoring") 


\section{Scale Reliability Analysis}

The groups of items measuring aspects of multicultural relationships were analyzed for internal consistency using scale reliability analysis for both pretreatment and posttreatment data. DeVellis (2003) indicated that an alpha score above .70 suggests acceptable levels of internal consistency. Table 3 presents the Cronbach's alpha values, means, and standard deviations for the five scales.

Table 3: Descriptive Statistics for the Scales in the Student Multicultural Relations Survey $(\mathrm{N}=54)$

\begin{tabular}{lcccccc}
\hline \multirow{2}{*}{ Scale } & \multicolumn{3}{c}{ Pretreatment Results } & \multicolumn{3}{c}{ Posttreatment Results } \\
\cline { 2 - 7 } & $\mathrm{\alpha}$ & $M$ & $S D$ & $\mathrm{a}$ & $M$ & $S D$ \\
\hline Multicultural Relationships & .79 & 3.90 & 0.62 & .86 & 4.09 & 0.69 \\
Actions of Multiculturalism & .84 & 4.13 & 0.68 & .82 & 4.10 & 0.69 \\
Awareness of Multiculturalism & .70 & 4.05 & 0.48 & .72 & 4.13 & 0.54 \\
Skills of Multicultural Education & .70 & 4.06 & 0.60 & .70 & 4.12 & 0.53 \\
Student Interactions & .17 & - & - & .12 & - & - \\
\hline
\end{tabular}

Three of the five scales required special treatment. The Awareness of Multiculturalism Scale was comprised of eight Survey Items (6, 10, 20,21, 22, 24, 25, and 26) and yielded a Cronbach's alpha of .69 for the pretreatment survey results. With Item 21 removed from the scale, an acceptable alpha value (.70) was achieved. For the posttreatment survey results, the Cronbach's alpha was acceptable with Item 21 included in the scale. However, to keep both pretreatment and posttreatment scales equivalent, Item 21 was removed from both scales. The Skills of Multicultural Education Scale comprised five Survey Items $(8,11,12,13$, and 14) and yielded a Cronbach's alpha of .68 for both the pretreatment and posttreatment survey results. With Item 8 removed from the scales, an acceptable alpha value (.70) was achieved. Therefore, Item 8 was removed from both the pretreatment and posttreatment scales. The Student Interactions Scale comprised Survey Items 9 and 23. For the purposes of determining scale reliability, Survey Item 9 was reverse coded because it had a negative valence. The internal consistency of the scales, both pretreatment and posttreatment, was unacceptable. Therefore, no Student Interactions scales were created. Items 9 and 23 were treated as separate variables in subsequent inferential statistical analysis.

According to the mean scores of the four reliable scales, students generally have positive perceptions about multicultural relations. All but one mean score were above the numerical value for the response of agree (i.e., 4), and the one mean score (pretreatment multicultural relations) below 4 (or agree) is a mean of 3.90 .

\section{Inferential Statistics Addressing the Research Questions}

A series of nine repeated measures $t$ tests was run to answer the first research question, "Does 4 months of participation in the CAC significantly change students' attitudes on multicultural relations, as measured by the Student Multicultural Relations Survey?" The results appear in Table 4. 
Baltes, Hernandez, \& Collins, 2015

Table 4: T Test Results Comparing Pretreatment and Posttreatment Student Attitudes

\begin{tabular}{|c|c|c|c|c|c|c|}
\hline \multirow{2}{*}{\multicolumn{2}{|c|}{ Scale or Variable }} & \multirow{3}{*}{$\frac{d f}{53}$} & \multirow{3}{*}{$\frac{t}{-2.74}$} & \multirow{3}{*}{$\frac{p}{0.008}$} & \multicolumn{2}{|c|}{$M(S D)$} \\
\hline & & & & & \multirow{2}{*}{$\frac{\text { Pretreatment }}{3.90(0.62)}$} & \multirow{2}{*}{$\frac{\text { Posttreatment }}{4.09(0.69)}$} \\
\hline 1 & Multicultural Relationships & & & & & \\
\hline 2 & Actions of Multiculturalism & 53 & 0.55 & 0.587 & $4.14(0.67)$ & $4.10(0.71)$ \\
\hline 3 & Awareness of Multiculturalism & 53 & -1.03 & 0.307 & $4.05(0.49)$ & $4.14(0.55)$ \\
\hline 4 & Skills of Multicultural Education & 53 & -1.45 & 0.152 & $4.06(0.60)$ & $4.21(0.53)$ \\
\hline 5 & $\begin{array}{l}\text { Q7: Students "hang out" with } \\
\text { students of same cultural } \\
\text { background }\end{array}$ & 53 & 0.22 & 0.83 & $3.61(0.92)$ & $3.57(0.92)$ \\
\hline 6 & $\begin{array}{l}\text { Q8: Students mix easily with } \\
\text { students from different cultural } \\
\text { backgrounds }\end{array}$ & 53 & -2.84 & 0.006 & $3.57(0.90)$ & $3.94(0.83)$ \\
\hline 7 & $\begin{array}{l}\text { Q9: Students are afraid of students } \\
\text { from different cultural backgrounds } \\
\text { that theirs }\end{array}$ & 53 & 1.7 & 0.096 & $2.31(0.84)$ & $2.02(1.06)$ \\
\hline 8 & $\begin{array}{l}\text { Q21: Various ethnic groups raise } \\
\text { their children differently }\end{array}$ & 53 & -1.75 & 0.086 & $4.06(0.76)$ & $4.26(0.65)$ \\
\hline 9 & $\begin{array}{l}\text { Q23: Having students from different } \\
\text { ethnic groups in classes is not a } \\
\text { problem }\end{array}$ & 53 & -0.83 & 0.41 & $4.09(0.88)$ & $4.20(0.88)$ \\
\hline
\end{tabular}

Only two comparisons yielded significant results. Students' attitudes on Multicultural Relationships were significantly more positive after 4 months of participation in the CAC, $t(53)=-2.74, p<.01$. Additionally, students believed they mix more easily with students from different cultural backgrounds after 4 months of participation in the CAC, $t(53)=-2.84, p<.01$. The mean differences between pretreatment and posttreatment attitudes on two other survey items (i.e., Item 9: Students are afraid of students from different cultural backgrounds than theirs, and Item 21: Various ethnic groups raise their children differently) were approaching significance, $p=.096$ and $p=.086$, respectively. Because the mean score of the posttreatment responses on Item 9 was lower than the pretreatment responses, the results indicate that after 4 months of participation in the CAC, students are less afraid of students from different cultural backgrounds than their own; however, not significantly so.

A second set of repeated measures $t$-test analyses was conducted on Survey Item 27, which went beyond exploring student attitudes; Item 27 deals with action-Do students talk to or mix with students of other cultural groups daily, during before and after school extracurricular activities, and socially outside the school setting. Two of the three $t$ tests were statistically significant-after 4 months of participation in the CAC, students reported increased talking to and mixing with students from other cultural groups (a) daily in class, $t(51)=-2.61, p<.05$, and (b) during before and after school extracurricular activities, $t(51)=-2.39, p<.05$.

With the evidence from the $t$ tests, the answer to Research Question 1 is that 4 months of participation in the CAC does change students' attitudes by raising their scores on one multicultural relations scale and three single-item variables.

To answer the second research question, a series of nine multiple regression analyses was run regressing multicultural relations change scores onto the two demographic variables of gender and 
ethnicity. Change scores were calculated by subtracting the pretreatment survey response values from the posttreatment survey response values. The ethnicity variable was dummy coded to yield two variables-African American and European American. The baseline ethnicity was "Other," and included all ethnicities other than African American or European American. Table 5 displays the results of the multiple regression calculations.

The results indicated that only one multiple regression model was significant. Being African American was a significant predictor of change in actions of multiculturalism, $F(3,50)=5.37, p<.01$, $R^{2}=.24$, adjusted $R^{2}=.20$. On average, African American students scored $0.91(B)$ scale points higher than students of other ethnicities as a result of participation in the CAC, which represents almost one full category change on the strongly-disagree-to-strongly-agree Likert scale.

Although the omnibus model regressing the Multicultural Relationship Scale onto the predictor variables was only approaching significance, $F(3,50)=1.86, p=.15$, one predictor was significant nonetheless. On average, European American students scored 0.64 (B) scale points lower than students of other ethnicities as a result of participation in the CAC, which represents just over a half category change on the strongly-disagree-to-strongly-agree Likert scale. This finding is the opposite of the intent of the CAC collaborative, which seeks to foster improved multicultural attitudes and relations. However, because the omnibus model was not significant, no individual predictor within the model can be treated as significant, even if the $t$-test calculation for a single predictor appears to be significant. 
Table 5: Results of Ordinary Least Squares Regression Analyses for Multicultural Relations Change Scores

Criterion Variable /

Predictor Variable

\begin{tabular}{llll}
$B$ & $S E B$ & $B$ & $t$ \\
\hline
\end{tabular}

Change in Multicultural Relationships

$$
R=.32, R^{2}=.10, \text { adjusted } R^{2}=.05, F(3,50)=1.86, p=.15
$$

$\begin{array}{lcccc}\text { Gender } & -0.05 & 0.15 & -0 & -0.30 \\ \text { Ethnicity } & & & & \\ \quad \text { African American } & -0.41 & 0.33 & -0.30 & -1.25 \\ \text { European American } & -0.64 & 0.31 & -0.60 & -2.09^{*}\end{array}$

Change in Actions of Multiculturalism

$$
R=.49, R^{2}=.24 \text {, adjusted } R^{2}=.20, F(3,50)=5.37, p<.01
$$

$\begin{array}{lcccc}\text { Gender } & 0.09 & 0.15 & 0.07 & 0.57 \\ \text { Ethnicity } & & & & \\ \quad \text { African American } & 0.91 & 0.34 & 0.66 & 2.71^{* *} \\ \quad \text { European American } & 0.28 & 0.32 & 0.22 & 0.91\end{array}$

Change in Awareness of Multiculturalism

$$
R=.26, R^{2}=.07 \text {, adjusted } R^{2}=.01, F(3,50)=1.19, p=.33
$$

$\begin{array}{lcccc}\text { Gender } & 0.12 & 0.18 & 0.09 & 0.64 \\ \text { Ethnicity } & & & & \\ \quad \text { African American } & -0.04 & 0.40 & -0 & -0.10 \\ \text { European American } & -0.34 & 0.38 & -0.30 & -0.91\end{array}$

Change in Skills of Multicultural Education

$$
R=.12, R^{2}=.01 \text {, adjusted } R^{2}=-.05, F(3,50)=.23, p=.88
$$

$\begin{array}{lllll}\text { Gender } & -0.06 & 0.22 & -0 & -0.29 \\ \text { Ethnicity } & & & & \\ \quad \text { African American } & -0.10 & 0.48 & -0.10 & -0.21 \\ \text { European American } & -0.26 & 0.45 & -0.20 & -0.58\end{array}$

Q7 Students "Hang Out" With Students of Same Cultural Background $R=.26, R^{2}=.07$, adjusted $R^{2}=.01, F(3,50)=1.24, p=.31$

$\begin{array}{lllll}\text { Gender } & 0.33 & 0.37 & 0.12 & 0.89\end{array}$

Ethnicity

$\begin{array}{lllll}\text { African American } & 0.58 & 0.81 & 0.19 & 0.72\end{array}$

$\begin{array}{lllll}\text { European American } & -0.08 & 0.76 & -0 & -0.10\end{array}$ 


\begin{tabular}{|c|c|c|c|c|}
\hline $\begin{array}{l}\text { Criterion Variable / } \\
\text { Predictor Variable }\end{array}$ & $B$ & $S E B$ & $B$ & $t$ \\
\hline \multicolumn{5}{|c|}{$\begin{array}{l}\text { Q8 Students Mix Easily With Students From Different Cultural Backgrounds } \\
\qquad R=.16, R^{2}=.03 \text {, adjusted } R^{2}=-.03, F(3,50)=0.43, p=.73\end{array}$} \\
\hline Gender & -0.32 & 0.29 & -0.20 & -1.12 \\
\hline \multicolumn{5}{|l|}{ Ethnicity } \\
\hline African American & $<0.01$ & 0.63 & $<.010$ & $<.010$ \\
\hline European American & -0.10 & 0.59 & -0.10 & -0.17 \\
\hline \multicolumn{5}{|c|}{$\begin{array}{l}\text { Q9 Students Are Afraid of Students From Different Cultural Backgrounds } \\
\text { Than Theirs } \\
\qquad R=.14, R^{2}=.02 \text {, adjusted } R^{2}=-.04, F(3,50)=0.33, p=.81\end{array}$} \\
\hline Gender & 0.34 & 0.39 & 0.13 & 0.88 \\
\hline \multicolumn{5}{|l|}{ Ethnicity } \\
\hline African American & -0.17 & 0.85 & -0.10 & -0.19 \\
\hline European American & -0.20 & 0.80 & -0.10 & -0.25 \\
\hline \multicolumn{5}{|c|}{$\begin{array}{l}\text { Q21 Various Ethnic Groups Raise Their Children Differently } \\
R=.06, R^{2}=<.01 \text {, adjusted } R^{2}=-.06, F(3,50)=0.05, p=.99\end{array}$} \\
\hline Gender & -0.07 & 0.26 & -0 & -0.25 \\
\hline \multicolumn{5}{|l|}{ Ethnicity } \\
\hline African American & -0.18 & 0.57 & -0.10 & -0.32 \\
\hline European American & -0.15 & 0.54 & -0.10 & -0.29 \\
\hline \multicolumn{5}{|c|}{$\begin{array}{l}\text { Q23 Having Students From Different Ethnic Groups in Classes Is } \\
\text { Not a Problem } \\
\qquad \begin{array}{l}R=.10, R^{2}=<.01, \text { adjusted } R^{2}=-.05, F(3,50)=0.15, p=.93\end{array}\end{array}$} \\
\hline Gender & 0.14 & 0.30 & 0.07 & 0.48 \\
\hline \multicolumn{5}{|l|}{ Ethnicity } \\
\hline African American & -0.13 & 0.66 & -0.10 & -0.20 \\
\hline European American & -0.2 & 0.62 & -0.10 & -0.33 \\
\hline
\end{tabular}

${ }^{*} p<.05 .{ }^{* *} p<.01$.

With only one of nine multiple regression models yielding significant results and only one of three predictors being statistically significant within that model, the answer to Research Question 2 is that neither gender nor ethnicity is a good predictor of changes in students' attitudes on multicultural relations after 4 months of participation in the CAC. 


\section{Summary}

The survey data showed that twice as many females than males completed usable surveys, with slightly over three times as many European American survey completers as African American survey completers. Of the five scales from the Student Multicultural Relations Survey, four had sufficient internal consistency ( $\alpha=.70$ to $\alpha=.86$ ) among the items to be used in further analysis. Single items, which were excluded from scales, were analyzed independently.

Key findings were that students' attitudes on (a) multicultural relationships and (b) their ability to mix more easily with students from different cultural backgrounds was significantly more positive after 4 months of participation in the CAC. Additionally, students talked to and mixed with students of other cultural groups both daily in class and before and after school during extracurricular school activities. Finally, being an African American student participating in the CAC for a 4-month period was a significant predictor of change in actions of multiculturalism.

\section{Research Question 1}

To determine whether 4 months of participation in the CAC had changed students' attitudes on multicultural relations, survey data were collected at two points in time: September 2012 and January 2013. In September, participants had just entered the CAC, and these data, collected prior to participation in the CAC, were considered pretreatment. September survey responses were matched with the January survey responses, after participants had participated in the CAC (the treatment) for 4 months.

The $t$ test analyses determined two significant differences in pretreatment-posttreatment attitudes. Students' attitudes significantly improved on (a) multicultural relationships and (b) their belief in their ability to mix more easily with students of different cultural backgrounds. These two findings are supported by the intergroup contact theory literature (Baltes, 1996; Crystal, Killen, \& Ruck, 2008; Gaunt, 2011; Ruck, Park, Killen, \& Crystal, 2011; Slavin \& Cooper, 1999; Stathi, Crisp, \& Hogg, 2011), which posits that intergroup contact promotes cross-race relationships. The Multicultural Relationships Scale encompasses the attitudes of the entire community as viewed from the students' point of view. It reflects the students' attitudes towards different cultural groups, as well as their perceptions of the attitudes of their friends and their parents.

Although, only two of the nine $t$ test comparisons yielded significant results. Two other comparisons were not significant, but were approaching significance, which might be due to the small sample size of 54, making it difficult for the $t$ test to detect small effects. Additionally, with only 4 months of participation in the CAC, the full effect of the treatment may not have been realized. If data were collected after students participated in the CAC for 1 full year (or longer), greater attitude changes may have resulted.

Finally, two significant $t$ tests provided support that the significant improvement in students' attitudes with respect to multicultural relations was real. Results of the statistical analysis of the data for survey question 27 showed that students significantly increased the frequency of their interactions of talking to and mixing with students of other cultural groups on a daily basis in class and in before and after school extracurricular activities. The students' change in attitudes was translated into action, if only within the school setting (i.e., in class and extracurricular activities centered around school).

With respect to outside the school setting, Figures 1 and 2 showed that within the last 12 months, students have had friends from a different culture in their homes and have worked on a school and 
community project with students of other cultures. After only 4 months of exposure to the CAC, students' attitudes and behaviors have changed towards students from other cultural groups.

\section{Research Question 2}

Research Question 2 asked if gender or ethnicity could predict a change in students' attitudes on multicultural relations after 4 months of participation in the CAC. In order to answer this research question, data were collected in the first 2 weeks of a student's entry into the CAC and again after 4 months enrollment and attendance (i.e., treatment). The pretreatment scores were subtracted from the posttreatment scores to determine change scores. The change scores data from 54 participants were regressed onto three variables-gender and two ethnicity variables, African American and European American—using multiple regression.

The results of the analysis showed that only one regression model, change in actions of multiculturalism, was statistically significant, and only one predictor (African American students) within the model was significant. Actions of multiculturalism involve more than just attitudes on multicultural relationships. The next step beyond students having more positive attitudes with respect to multicultural relationships is the students' declaration of a willingness to act on those attitudes (i.e., actions on multiculturalism). In other words, the student looks for or would welcome opportunities to be immersed in multicultural activities. The final stage occurs when students actually take steps to follow through with action. Talking to and mixing with students from a different cultural group than one's own translates one aspect of the Actions of Multiculturalism Scale from intent to action. Evidence from two $t$ test analyses—students talking to and mixing with students from different cultural groups (a) daily in the classroom and (b) during before and after school extracurricular activities-demonstrates that students have put their changed attitudes into practice, if even just in a small way.

\section{Recommendations and Conclusions}

This study's findings should be interpreted cautiously due to the small sample size and the limited statistically significant results. However, the $t$ test results do provide preliminary evidence that the policies and practices implemented at the CAC are increasing cultural awareness and improving harmonious culture within a school setting of a diverse student body. Some attitudes were significantly more positive, and some behaviors relating to talking to and mixing with students of other cultural groups increased significantly.

Because of the positive results, further study of how the CAC structures enrollees' intercultural experiences throughout the curriculum and during the school day would be beneficial so that other schools or collaboratives could duplicate this model and strive to achieve similar results. In other words, school systems that have less than ideal cross-cultural harmony can use the findings of this study to support a change in their current practices to adopt practices more akin to those of the CAC studied in this research project.

Because the purpose of the CAC is to encourage students to learn about themselves and each other and help them build friendships that can facilitate good racial and cultural relations, one might reasonably expect a change in students' attitudes on multicultural relations after their participation in the CAC. Students did increase their acceptance of others, as demonstrated by more positive attitudes and increased cross-cultural interactions. These students will now be able to take their new 
attitudes and values with them into the workforce after they graduate. They may serve as models of appropriate acceptance, reducing interracial tension, resentment, and violence.

Moreover, the full benefits of the CAC, in terms of its ability to improve attitudes and encourage positive cross-cultural interactions, may not have been fully realized because the data were based on students' participation in the CAC "experience" for only 4 months-a relatively short period of time in light of the full 3 years most students will spend attending the CAC. This research study may have only detected preliminary changes in attitudes and behaviors, and more extensive changes might occur after continuous exposure to participation in the CAC.

\section{References}

Allport, G. (1958). The nature of prejudice. Garden City, NY: Doubleday.

Baltes, B. (1996). Personal life experiences and work-related factors associated with educators' approaches to multicultural education. In S. Sarsar (Ed.), Education for leadership and social responsibility (pp. 129-137). West Long Branch, NJ: Center for the Study of Public Issues.

Baltes, B. (1998). Creative teaching: Can we be multicultural? In H. E. Klein (Ed.). Creative Teaching, Act 1 (pp. 55-57). Madison, WI: Omni Press.

Bazron, B., Osher, D., \& Fleishmann, S. (2005). Creating culturally responsive schools. Educational Leadership, 63, 83-84.

Carter, R. B., \& Vuong, T. K. (1997). Unity through diversity: Fostering cultural awareness. Professional School Counseling, 1, 47-49.

Chen, G. M., \& Starosta, W. J. (1996). Intercultural communication competence: A synthesis. Communication Yearbook, 19, 353-384.

Crystal, D. S., Killen, M., \& Ruck, M. (2008). It is who you know that counts: Intergroup contact and judgments about race-based exclusion. British Journal of Developmental Psychology, 26, 5170. doi:10.1348/026151007X198910

Deardorff, D. K. (2004). In search of intercultural competence. International Educator, 13, 1315. doi:772375861

DeLong, M., Geum, K., Gage, K., McKinney, E., Medvedev, K., \& Park, J. (2011). Cultural exchange: Evaluating an alternative model in higher education. Journal of Studies in International Studies, 15, 41-56. doi:10.1177/1028315309334619

DeVellis, R. F. (2003). Scale development: Theory and applications (2nd ed., Vol. 26, Applied social research methods series). Thousand Oaks, CA: Sage.

Federal Bureau of Investigation. (2009). Hate crimes statistics. Washington, DC: Department of Justice. Retrieved from http://www2.fbi.gov/ucr/hc2009/index.html

Federal Bureau of Investigation. (2010). Hate crimes statistics. Washington, DC: Department of Justice. Retrieved from http://www.fbi.gov/about-us/cjis/ucr/hate-crime/2010

Gaunt, R. (2011). Effects of intergroup conflict and social contact on prejudice: The mediating role of stereotypes and evaluations. Journal of Applied Social Psychology, 41, 1340-1355. doi:10.1111/j.1559-1816.2011.00762.x

Hansell, L. (2000). Putting contact theory into practice: Using the PARTNERS Program to develop intercultural competence. Electronic Magazine of Multicultural Education, 2. Retrieved from http://www.eastern.edu/publications/emme/2000fall/hansell.html 
Liaw, M. (2006). E-learning and the development of intercultural competence. Language Learning and Technology, 10, 49-64. Retrieved from http://lt.msu.edu/vol10num3/liaw/default.html

Loukaitou-Sideris, A. (2003). Children's common ground: A study of intergroup relations among children in public settings. Journal of American Planning Association, 69, 130-141. doi:10.1080/01944360308976302

Maddox, M. (2012, March 27). Racist note found at Seaholm High School aimed at African American staff and a student. ABC 7 Action News. Retrieved from http://www.wxyz.com/news/racistnote-found-at-seaholm-high-school-aimed-at-african-american-staff-and-a-student

Pettigrew, T. (1998). Intergroup contact theory. Annual Review of Psychology, 49, 65-85. doi:10.1146/annurev.psych.49.1.65

Pettigrew, T., \& Tropp, L. (2006). A meta-analytic test and reformation of intergroup contact theory. Journal of Personality and Social Psychology, 90, 751-783. doi:10.1037/0022-3514.90.5.751

Quappe, S., \& Cantatore, G. (2005). What is cultural awareness, anyway? How do I build it? Where growing minds go global. Retrieved from www.culturosity.com/pdfs/What\%20is\%20Cultural\%20Awareness.pdf

Reich, S. M., \& Reich, J.A. (2006). Cultural competence in interdisciplinary collaborations: A method for respecting diversity in research partnerships. American Journal of Community Psychology, 38, 51-62. doi:10.1007/s10464-9064-1

Richards, H., Brown, H., \& Forde, T. (2007). Addressing diversity in schools: Culturally responsive pedagogy. Teaching Exceptional Children, 39, 64-68.

Richardson, T. L. (2012). Programming tips from HBCUs for engaging African-American students at predominantly White institutions (PWIs). Campus Activities Programming, 45, 32-33.

Riskowski, J. L., \& Olbricht, G. (2010). Student views of diversity through a multicultural mathematics activity: Viewing transformation during the middle school years. Multicultural Education, 17, 2-12. (ERIC Document Reproduction Service No. EJ887768)

Rothfarb, S. H. (1992). Perceptions of intergroup relations in secondary schools of Dade County Public Schools: Results of the Student Multicultural Relations Survey. Miami, FL: Dade County Public Schools. (ED 408 373).

Rothman, S., Lipset, S., \& Nevitte, N. (2003). Racial diversity reconsidered. Public Interest, 151, 2550.

Ruck, M. D., Park, H., Killen, M., \& Crystal, D. S. (2011). Intergroup contact and evaluations of racebased exclusion in urban minority children and adolescents. Journal of Youth and Adolescence, 40, 633-643. doi:10.1007/s10964-010-9600-z

Schuitema, J., \& Veugelers, W. (2011). Multicultural contacts in education: A case study of an exchange project between different ethnic groups. Educational Studies, 37, 101-114. doi:10.1080/03055691003729252

Slavin, R., \& Cooper, R. (1999). Summary of improving intergroup relations: Lessons learned from cooperative learning programs. Journal of Social Issues, 55, 647-664. doi:10.1111/00224537.00140

Stathi, S., Crisp, R. J., \& Hogg, M. A. (2011). Imagining intergroup contact enables member-to-group generalization. Group Dynamics: Theory, Research, and Practice, 15, 275-284. doi:10.1037/a0023752 
United States Census Bureau. (2012). Percentage of public schools reporting selected types of disciplinary problems at school by selected school characteristics: 2007 to 2008. Washington, DC: Author. Retrieved from http://www.census.gov

Woods, D. R., III. (2009). High school students' perceptions of the inclusion of multicultural education in a suburban school district (Doctoral dissertation). Retrieved from ProQuest Dissertations \& Theses: The Humanities and Social Sciences Collection. (AAT 3359586)

The Journal of Educational Research and Practice provides a forum for studies and dialogue that allows readers to better develop social change in the field of education and learning. Journal content may focus on educational issues of all ages and in all settings. It also presents peer-reviewed commentaries, book reviews, interviews of prominent individuals, and additional content. The objectives: We publish research and related content that examines current relevant educational issues and processes aimed at presenting readers with knowledge and showing how that knowledge can be used to impact social change in educational or learning environments. Additional content provides an opportunity for scholarly and professional dialogue regarding that content's usefulness in expanding the body of scholarly knowledge and increasing readers' effectiveness as educators. The journal also focuses on facilitating the activities of both researcher-practitioners and practitioner-researchers, providing optimal opportunities for interdisciplinary and collaborative thought through blogging and other communications.

Walden University Publishing: http://www.publishing.waldenu.edu 